\title{
An Extended-DORT method and its application in a cavity configuration
}

\author{
X. Y. Zhang, H. Tortel, A. Litman, J.-M. Geffrin \\ Aix-Marseille Université, CNRS, Centrale Marseille, Institut Fresnel UMR 7249, \\ Campus de St Jérôme, 13397 Marseille Cedex, France \\ $\mathrm{X}$. Y. Zhang is now with the Science and Technology on Electronic Information \\ Control Laboratory,Chengdu 610036, China \\ E-mail: amelie.litman@fresnel.fr
}

Submitted to: Inverse Problems

\begin{abstract}
In this work, we present an extension of the classical DORT method for time harmonic inverse medium scattering problems. This tool enables to estimate the shape of the unknown scatterers even if they are not necessarily point-like scatterers. This method is described and compared with the already existing sampling methods. Moreover, a mathematical derivation is provided for its validation, either in a freespace environment or in a metallic enclosure setup. Two-dimensional examples, based on measured data sets acquired in the circular microwave scanner developed at Institut Fresnel, are presented, which show that the method is computationally efficient and robust to noise.
\end{abstract}




\section{Introduction}

It is well known that electromagnetic scattering phenomenas are governed by two coupled integral equations [1]. The second-order Fredholm integral equation provides the field inside the scattering object, by fully taking into account for the multiple scattering interactions effects. The first-order Fredholm integral equation links the induced currents inside the target with the radiated fields that can be measured outside the object. If one is interested in recovering quantitative permittivity maps from the measured scattered field, the two sets of equation must be solved simultaneously. The inverse problem is then recast into an optimization in which the electromagnetic parameters are progressively recovered $[2,3,4,5]$, the ill-posedness nature of the problem being eventually reduced by an appropriate description of the unknowns [6] or by the use of regularization parameters [7]. Unfortunately, the computational burden associated to this kind of problem may grow rapidly.

In some applications, only a limited amount of information is required, as for example the footprint of the unknown target. In this framework of qualitative imagery, several methods, which only consider the observation equation, have been proposed. Among the different techniques yielding the shape of the scatterer, one can cite the diffraction tomography algorithm if the measurement is performed in farfield [8] or the back-propagation algorithm [2]. Several sampling methods have also been derived, such as the MUSIC method, the Linear Sampling Method (LSM), the Factorization Method (FM) or the subspace migration methods (SM). These methods have been successfully applied in many cases, for various types of data $(2 \mathrm{D}, 3 \mathrm{D}$, numerical data, experimental ones) and scatterers (metallic, dielectric, non-connected, ...) $[9,10,11,12,13,14,15,16,17,18,19]$. Finally, if one is only interested in the localization of point-like scatterers, the DORT method is particularly well suited [20].

In all these sampling methods, apart from [21], the multi-incidence multi-receiver transfer matrix is the main piece of information being processed. More specifically, its singular system is first extracted and, for any given point in the test domain, the shape indicator is then built thanks to a linear combination of the back-propagated singular vectors. It turns out that all these sampling methods are only differing in the weighting coefficients which are applied to each of the back-propagated singular vectors. The simplest algorithm is thus corresponding to the DORT method as only the first term is considered, but it is unfortunately only efficient for point-like scatterers. For extended scatterer, the MUSIC, the LSM, the FM method or some versions of the SM method may apply. But they all rely on the adequate choice of the external hyperparameters, either the dimension of the noise space for the first method, the Tikhonov regularization parameter for the second and the third methods, or on the weights which are selected in the SM methods. These parameters can be very difficult to determine, in particular when we are dealing with real data, even if theoretical rules may apply. If they are incorrectly selected, it can dramatically modify the reconstructed shape of the object. From these considerations, we came up with the idea of changing the weighting 
coefficients. We are thus proposing a natural weighting function, exempt from any kind of tuning parameter, which can be seen as an extension of the classical DORT method applicable not only to point-like scatterers but also to extended ones.

To assess the validity of this Extended-DORT shape indicator, we first analytically studied its convergence properties. To verify its robustness with respect to noise, we confronted it with experimental scattered fields. Instead of using the free-space measured fields of the Fresnel database [22], we took advantage of a more complicated setup, which corresponds to a circular metallic enclosure that we also have at our disposal at Institut Fresnel [23]. As the Green's function is changing with the boundary conditions, the singular system must also reflect the changes in the measurement environment. The various methods have thus been detailed in this specific environment and the convergence properties of the EDORT method have also been derived.

The paper is organised as follows. Section 2 describes the considered configurations, either the free-space case or the metallic enclosure setup. Section 3 and 4 are dedicated to the analytical derivation of the singular system of the multi-static transfer matrices and the back-propagated fields in these configurations. In Section 5, we present the Extended DORT method. Section 6 describes the experimental microwave setup and the data processing method followed in order to obtain valuable scattered fields data from the measurements. Comparisons of the results obtained with the various shape indicators are also displayed. Conclusions follow. A study of the convergence properties of the EDORT shape indicator for the free space configuration and the cavity configuration is performed in the Appendices.

\section{Configurations}

Let us consider the geometry of the two-dimensional inverse problems shown in Figure 1. The cavity configuration is also a cross-section of a multi-static microwave scanner under development in our laboratory $[6,23,24,25]$ which is further detailed in Section 6.1.

An unknown object contained in a circular zone $\Omega$, with radius $R_{\Omega}$ and center $O^{\prime}$, is illuminated by a set of incident electromagnetic fields generated by an array of $N$ antennas working at a single frequency (with a time dependency in $\exp (-j \omega t)$ ). These antennas are placed in the same horizontal plane, on a circle $\Gamma$, with radius $R_{\Gamma}$, centered on $O$. They are assumed to radiate a TM linearly polarized field and all the cylinders are supposed infinitely long along the $z$ direction, which renders possible a two-dimensional assumption. In the cavity configuration, a circular metallic enclosure $\Sigma$ is surrounding the measurement setup. This casing is such that $R_{\Sigma}=R_{\Gamma}+\frac{\lambda}{4}[26]$.

Each antenna is sequentially acting as an emitter and a receiver, providing at the end of the measurement procedure, a $N \times N$ multi-static measurement transfer matrix $\mathbb{K}=\left\{K_{r e}\right\}$, with $e, r=1, \cdots, N$, where $K_{r e}$ is the inner element response between the $e$-th emitter $E_{e}$ and the $r$-th receiver $R_{r}$. 

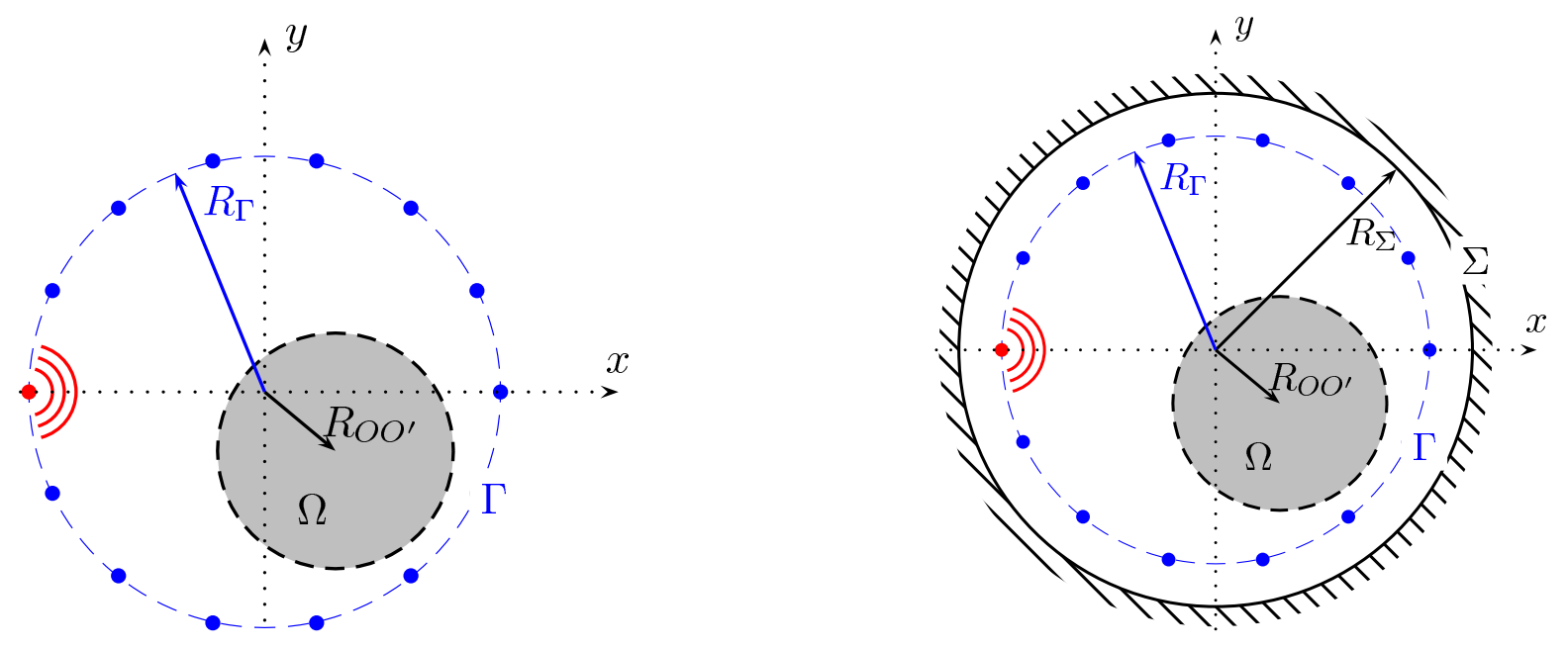

Figure 1. (Left) Free space and (Right) cavity configurations

\section{Singular system of the multi-static transfer matrices $\mathbb{K}$}

Many of the qualitative imaging methods which are currently available (DORT, Music, LSM, Factorization method, ...) are based on the SVD decomposition the scattering matrix $\mathbb{K}, u_{n}$ are corresponding to the left singular vectors of $\mathbb{K}$, and the singular values are noted $\sigma_{n}$.

\subsection{Free-space singular system}

In a free-space environment and in a complete configuration, analytical analysis [27] have provided closed-form expressions which show the direct correspondence between a point scatterer and a singular vector. Indeed, in free-space, the multi-static transfer matrix can be written as

$$
\mathbb{K}^{\text {free }}=\left[\mathbb{H}^{+}\left(\overrightarrow{O^{\prime} R}\right)\right]^{t} \mathbb{S}^{\prime} \mathbb{H}^{-}\left(\overrightarrow{O^{\prime} E}\right)
$$

where $\mathbb{S}^{\prime}$ corresponds to the scattering matrix of the target computed by taking into account its center $O^{\prime}$. This scattering matrix is diagonal if the scatterer is cylindrical, and reduces to a single value if the scatterer is small with respect to the wavelength. The propagation matrices $\mathbb{H}^{ \pm}$are defined in a free-space configuration as

$$
\mathbb{H}^{ \pm}\left(\overrightarrow{O^{\prime} M}\right)=\left(\begin{array}{ccccc}
H_{-n}\left(k \rho_{1}^{\prime}\right) e^{\mp j n \phi_{1}^{\prime}} & \cdots & H_{-n}\left(k \rho_{i}^{\prime}\right) e^{\mp j n \phi_{i}^{\prime}} & \cdots & H_{-n}\left(k \rho_{N}^{\prime}\right) e^{\mp j n \phi_{N}^{\prime}} \\
\vdots & & & & \vdots \\
H_{0}\left(k \rho_{1}^{\prime}\right) & \cdots & H_{0}\left(k \rho_{i}^{\prime}\right) & \cdots & H_{0}\left(k \rho_{N}^{\prime}\right) \\
\vdots & & & & \vdots \\
H_{n}\left(k \rho_{1}^{\prime}\right) e^{ \pm j n \phi_{1}^{\prime}} & \cdots & H_{n}\left(k \rho_{i}^{\prime}\right) e^{ \pm j n \phi_{i}^{\prime}} & \cdots & H_{n}\left(k \rho_{N}^{\prime}\right) e^{ \pm j n \phi_{N}^{\prime}}
\end{array}\right)
$$

where $H_{p}$ is the first-kind Hankel function of order $p, k$ is the embedding material wavenumber, and the values $\left(\rho_{i}^{\prime}, \phi_{i}^{\prime}\right)$ correspond to the polar coordinates of the family vectors $\overrightarrow{O^{\prime} M}=\left\{{\overrightarrow{O^{\prime} M}}_{i}, i=1, \cdots, N\right\}$, where $O^{\prime}$ is the center of the scatterer. It can be shown 
that, in the case of a complete configuration, i.e., when the sources and the receivers completely surround the target zone, these matrices may be unitary matrices when a weak approximation is employed [27]. The multi-static transfer matrix can then be transformed into

$\mathbb{K}^{\text {free }}=\left[\mathbb{H}_{s}^{+}\left(\overrightarrow{O^{\prime} R}\right)\right]^{t}\left[\mathbb{D}^{\text {free }}\left(\overrightarrow{O^{\prime} R}\right)\right]^{1 / 2} \mathbb{S}^{\prime}\left[\mathbb{D}^{\text {free }}\left(\overrightarrow{O^{\prime} E}\right)\right]^{1 / 2} \mathbb{H}_{s}^{-}\left(\overrightarrow{O^{\prime} E}\right)$

where $\mathbb{H}_{s}^{ \pm}\left(\overrightarrow{O^{\prime} M}\right)=\left[\mathbb{D}^{\text {free }}\left(\overrightarrow{O^{\prime} M}\right)\right]^{1 / 2} \mathbb{H}^{ \pm}\left(\overrightarrow{O^{\prime} M}\right)$ are scaled unitary matrices with

$\mathbb{D}^{\text {free }}\left(\overrightarrow{O^{\prime} M}\right)=\left(\begin{array}{ccc}\sum_{p}\left|H_{-n}\left(k \rho_{p}^{\prime}\right)\right|^{2} & 0 & 0 \\ 0 & \sum_{p}\left|H_{0}\left(k \rho_{p}^{\prime}\right)\right|^{2} & 0 \\ 0 & 0 & \sum_{p}\left|H_{n}\left(k \rho_{p}^{\prime}\right)\right|^{2}\end{array}\right)$

\subsection{Cavity singular system}

In our current experiment, the propagation matrices must take into account the metallic enclosure. The singular system of $\mathbb{K}^{\text {cav }}$ has a more complicated expression but yields nevertheless the same behavior. Indeed, the multi-static transfer matrix can be written as

$$
\mathbb{K}^{c a v}=\left[\mathbb{F}^{+}(\overrightarrow{O R})\right]^{t} \mathbb{S} \mathbb{F}^{-}(\overrightarrow{O E})
$$

where $\mathbb{S}$ is the same scattering matrix as in Equation 1 except that this time, the scattering matrix is computed with respect to $O$, the center of the metallic casing. The propagation matrices $\mathbb{F}^{ \pm}$are now defined as

$$
\mathbb{F}^{ \pm}(\overrightarrow{O M})=\left(\begin{array}{ccccc}
f_{-n}\left(k \rho_{1}\right) e^{\mp j n \phi_{1}} & \cdots & f_{-n}\left(k \rho_{i}\right) e^{\mp j n \phi_{i}} & \cdots & f_{-n}\left(k \rho_{N}\right) e^{\mp j n \phi_{N}} \\
\vdots & & & & \vdots \\
f_{0}\left(k \rho_{1}\right) & \cdots & f_{0}\left(k \rho_{i}\right) & \cdots & f_{0}\left(k \rho_{N}\right) \\
\vdots & & & & \vdots \\
f_{n}\left(k \rho_{1}\right) e^{ \pm j n \phi_{1}} & \cdots & f_{n}\left(k \rho_{i}\right) e^{ \pm j n \phi_{i}} & \cdots & f_{n}\left(k \rho_{N}\right) e^{ \pm j n \phi_{N}}
\end{array}\right)
$$

where $(\rho, \phi)$ correspond to the polar coordinates of the family vectors $\overrightarrow{O M}$. The function $f_{p}$ reflects the boundary conditions $[28,29,26]$

$$
f_{n}(k \rho)=\frac{J_{n}(k \rho) Y_{n}\left(k R_{\Sigma}\right)-J_{n}\left(k R_{\Sigma}\right) Y_{n}(k \rho)}{J_{n}\left(k R_{\Sigma}\right)}
$$

To compare effectively the multi-static transfer matrices expressions, we exploit Graf's theorem [30] written in matrix form in order to introduce the scattering matrix $\mathbb{S}^{\prime}$ in Equation 5. Indeed, it can be shown that

$$
\mathbb{F}^{ \pm}(\overrightarrow{O M})=\mathbb{T}_{\mathbb{J}}^{ \pm}\left(\overrightarrow{O O^{\prime}}\right) \mathbb{F}^{ \pm}\left(\overrightarrow{O^{\prime} M}\right)
$$

where $\mathbb{T}_{\mathbb{J}}^{ \pm}\left(\overrightarrow{O O^{\prime}}\right)$ is a Toeplitz matrix such that

$$
\mathbb{T}_{\mathbb{J}}^{ \pm}\left(\overrightarrow{O O^{\prime}}\right)=\left(\begin{array}{ccccc}
\cdots & \cdots & \cdots & \cdots & \cdots \\
\cdots & J_{0}\left(k R_{0^{\prime}}\right) & J_{1}\left(k R_{0^{\prime}}\right) e^{\mp j \theta_{0^{\prime}}} & J_{2}\left(k R_{0^{\prime}}\right) e^{\mp 2 j \theta_{0^{\prime}}} & \cdots \\
\cdots & J_{-1}\left(k R_{0^{\prime}}\right) e^{ \pm j \theta_{0^{\prime}}} & J_{0}\left(k R_{0^{\prime}}\right) & J_{1}\left(k R_{0^{\prime}}\right) e^{\mp j \theta_{0^{\prime}}} & \cdots \\
\cdots & J_{-2}\left(k R_{0^{\prime}}\right) e^{ \pm 2 j \theta_{0^{\prime}}} & J_{-1}\left(k R_{0^{\prime}}\right) e^{ \pm j \theta_{0^{\prime}}} & J_{0}\left(k R_{0^{\prime}}\right) & \cdots \\
\cdots & \cdots & \cdots & \cdots & \cdots
\end{array}\right)
$$


where $\left(R_{O^{\prime}}, \theta_{O^{\prime}}\right)$ correspond to the polar coordinate of $O^{\prime}$ with respect to $O$. The multistatic transfer matrix can now be written as

$\mathbb{K}^{\text {cav }}=\left[\mathbb{F}^{+}\left(\overrightarrow{O^{\prime} R}\right)\right]^{t} \mathbb{S}^{\prime} \mathbb{F}^{-}\left(\overrightarrow{O^{\prime} E}\right) \quad$ with $\quad \mathbb{S}^{\prime}=\left[\mathbb{T}_{\mathbb{J}}^{ \pm}\left(\overrightarrow{O O^{\prime}}\right)\right]^{t} \mathbb{S} \mathbb{T}_{\mathbb{J}}^{ \pm}\left(\overrightarrow{O O^{\prime}}\right)$

It can also be shown that, in the case of a complete configuration, i.e., when the sources and the receivers completely surround the target zone, the propagation matrices $\mathbb{F}^{ \pm}\left(\overrightarrow{O^{\prime} R}\right)$ and $\mathbb{F}^{ \pm}\left(\overrightarrow{O^{\prime} E}\right)$, when properly scaled, may be considered as unitary matrices when a weak approximation is employed (see Appendix A). The multi-static transfer matrix can finally be written as

$$
\mathbb{K}^{c a v}=\left[\mathbb{F}_{s}^{+}\left(\overrightarrow{O^{\prime} R}\right)\right]^{t}\left[\mathbb{D}^{c a v}\left(\overrightarrow{O^{\prime} R}\right)\right]^{1 / 2} \mathbb{S}^{\prime}\left[\mathbb{D}^{c a v}\left(\overrightarrow{O^{\prime} E}\right)\right]^{1 / 2} \mathbb{F}_{s}^{+}\left(\overrightarrow{O^{\prime} E}\right)
$$

where $\mathbb{D}^{\text {cav }}$ is defined in (Eq. A.11).

\section{Back-propagated fields}

As it is difficult to derive generic rules for the singular vectors and their associated singular values, we will focus only in that section on the specific case of a circular scatterer. Indeed, in that case, close-form formulas exist for the scattering matrix $\mathbb{S}^{\prime}$, showing that this matrix is diagonal $[27,31,32]$.

\subsection{Back-propagated singular vectors}

In free-space, when a weak approximation is employed and for a circular scatterer, the singular vectors $\mathbb{U}^{\text {free }}$ of $\mathbb{K}^{\text {free }}$ will be represented by the propagation matrix $\left[\mathbb{H}_{s}^{+}\left(\overrightarrow{O^{\prime} R}\right)\right]^{t}$. Similarly, in the cavity configuration, for a circular scatterer, the singular vectors $\mathbb{U}^{c a v}$ of $\mathbb{K}^{\text {cav }}$ are nothing but the propagation matrix $\left[\mathbb{F}_{s}^{+}\left(\overrightarrow{O^{\prime} R}\right)\right]^{t}$.

The back-propagated fields are obtained by computing the quantity $\left\langle G \mid u_{n}\right\rangle_{\Gamma}$ where $\langle\mid\rangle_{\Gamma}$ corresponds to the scalar product on the probing line $\Gamma$ and $G$ is the Green's function of the current experiment. For the two configurations at hand, we have

$$
\begin{aligned}
& G^{\text {free }}\left(\vec{r} \in \Omega ; \vec{r}^{\prime} \in \Gamma\right)=\sum_{p} H_{p}\left(k \rho^{\prime}\right) J_{p}(k \rho) e^{i p\left(\theta^{\prime}-\theta\right)} \\
& G^{c a v}\left(\vec{r} \in \Omega ; \vec{r}^{\prime} \in \Gamma\right)=\sum_{p} f_{p}\left(k \rho^{\prime}\right) J_{p}(k \rho) e^{i p\left(\theta^{\prime}-\theta\right)}
\end{aligned}
$$

Therefore, taking into account the orthogonality properties of the propagation matrices, the back-propagated fields will be provided by,

$$
\begin{aligned}
\left\langle G^{\text {free }} \mid u_{n}\right\rangle_{\Gamma} & =\left\langle H_{n}\left(k \rho^{\prime}\right) e^{i n \theta^{\prime}} \mid u_{n}\right\rangle_{\Gamma} J_{n}(k \rho) e^{i n \theta} \\
\left\langle G^{\text {cav }} \mid u_{n}\right\rangle_{\Gamma} & =\left\langle f_{n}\left(k \rho^{\prime}\right) e^{i n \theta^{\prime}} \mid u_{n}\right\rangle_{\Gamma} J_{n}(k \rho) e^{i n \theta}
\end{aligned}
$$

In both cases, these fields contain terms in $J_{n}(k \rho)$ which provide information on the inner targets structure (Figure 2) as the support of such functions are either inside (for small index $n$ ) or outside (for large index $n$ ) of the scatterer support.

In particular, for a single small scatterer, if we back-propagate the vector $u_{n_{1}}$ which

corresponds to the leading singular value $\sigma_{n_{1}}$, the field will focus on the place where 


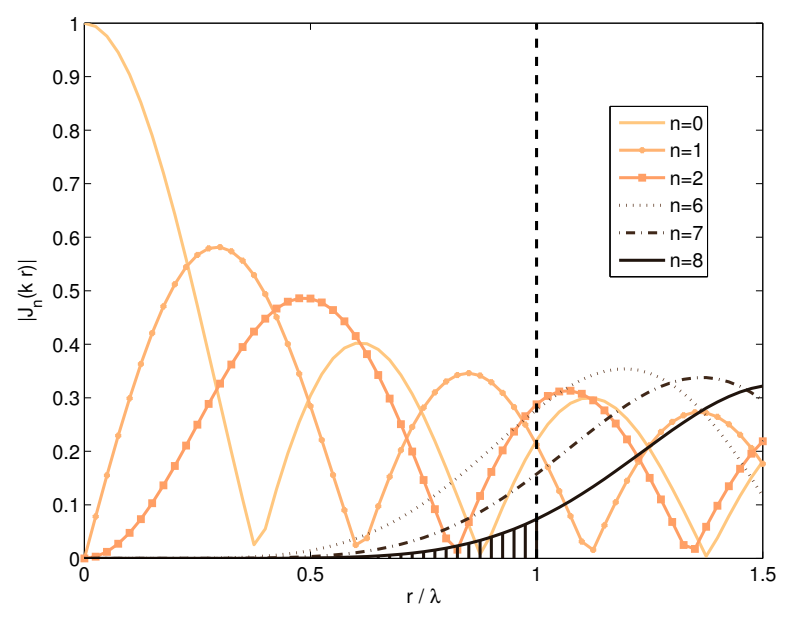

Figure 2. Amplitude of $J_{n}(k \rho)$ for various index $n$. The full vertical line corresponds to the case where $R_{\Omega}=\lambda$.

exists the associated point target. For extended targets, the one-to-one correspondence between the scatterer and the singular vector does not hold true anymore [33]. Thus the field generated by back-propagating a single $u_{n}$ can not focus on the entire extended target, but only on a limited part of it.

To get a better understanding of the singular vectors influence, we are considering the scattering by a dielectric cylinder $\left(\varepsilon_{r}=65+4 j\right)$ with a radius of $5 \mathrm{~cm}$ centered at $(10,-10) \mathrm{cm}$ in the cavity configuration (Figure 3$)$. Using a finite element software [24], we can easily construct the multi-static transfer matrix $\mathbb{K}^{\text {cav }}$ and calculate numerically the cavity Green's function $G^{c a v}\left(\vec{r}, \vec{r}^{\prime}\right)$, taking advantage of the reciprocity principle.

The modulus of the field patterns associated to the different $u_{n}^{\text {cav }}$ are shown in Figure 3. The singular vectors $u_{n}^{c a v}$ are here taken as unitary vectors, so all the square modulus of the field patterns have a finite and comparable range with each other. Besides, one can clearly see the changing process of the energy distribution, as for low indexes, the energy focus inside and around the target, while at high indexes, the energy tends to be outside. It is of interest to note that we can observe, in the first orders, not only some high energy points inside the scatterer but also at some "mirror" locations due to the various reflections at the metallic boundary.

\subsection{Behavior of the singular values}

As pointed out in Figure 3, the leading singular value (which corresponds to the first singular value when computed by a numerical SVD) does not provide a back-propagated field which necessarily corresponds to a $J_{0}$ pattern. It is therefore of interest to investigate the behavior of the singular values $\sigma_{n}$ for a fixed scatterer radius $R_{\Omega}$. We will consider here only the case of an impenetrable metallic circular scatterer. From (Eq. 3) or (Eq. 11), we can specify the singular value matrix of $\mathbb{K}$ as $\left[\mathbb{D}\left(\overrightarrow{O^{\prime} R}\right)\right]^{1 / 2} \mathbb{S}^{\prime}\left[\mathbb{D}\left(\overrightarrow{O^{\prime} E}\right)\right]^{1 / 2}$ where the scattering matrix of an impenetrable scatterer is a diagonal matrix whose 

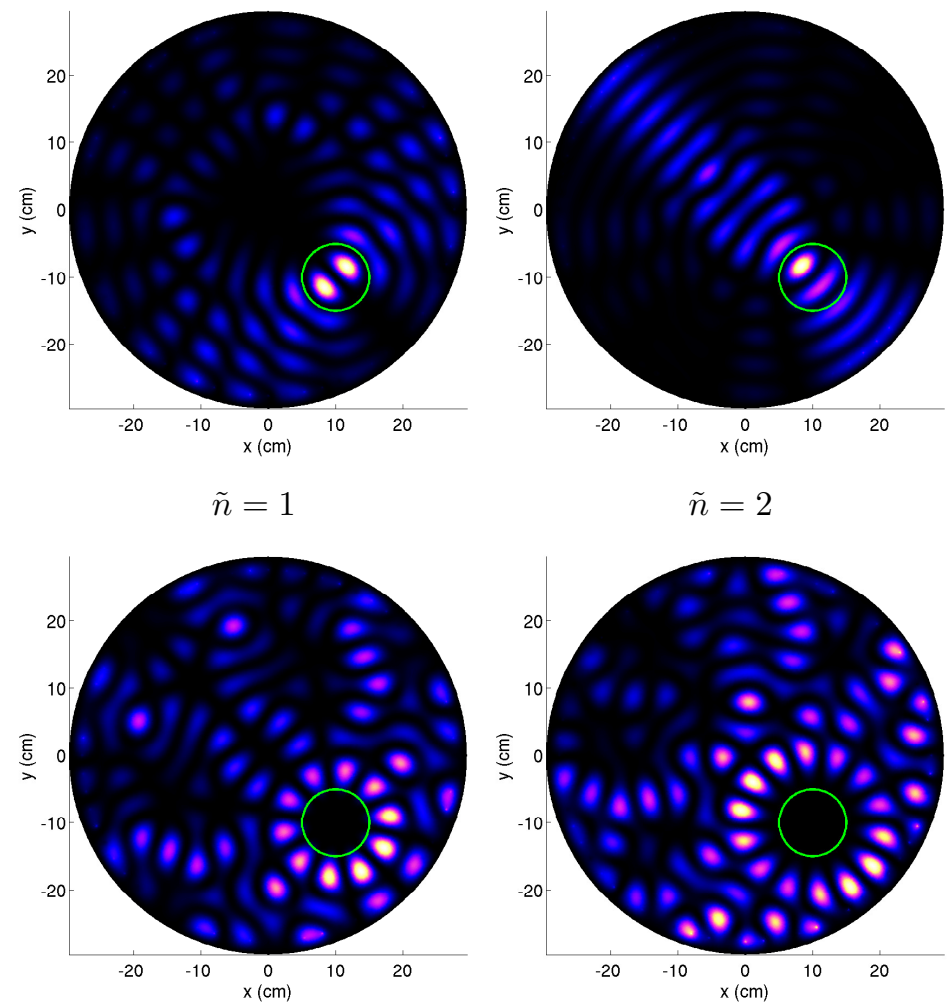

$\tilde{n}=10$

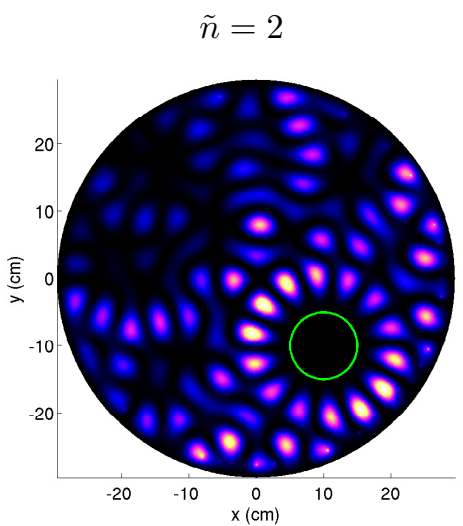

$\tilde{n}=15$
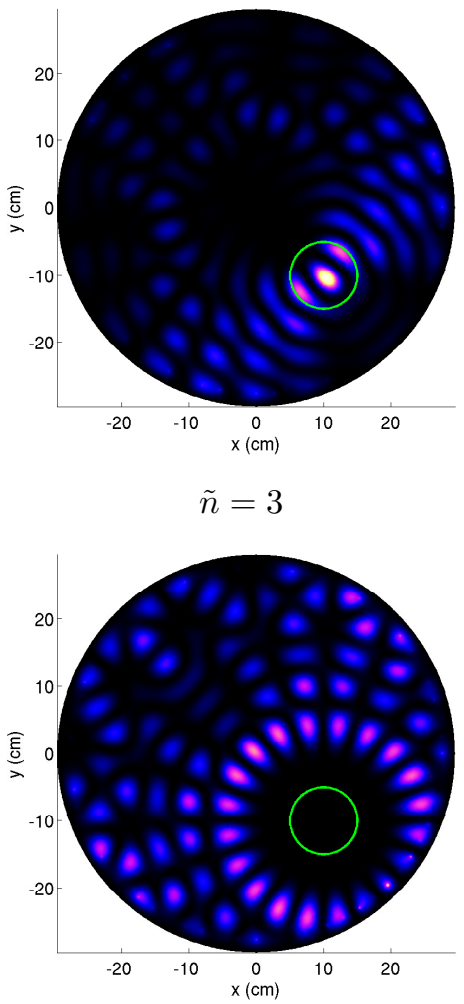

$\tilde{n}=20$

Figure 3. Amplitude of the different field patterns $\left|\left\langle G^{c a v} \mid u_{\tilde{n}}^{c a v}\right\rangle_{\Gamma}\right|^{2}$. The green contour corresponds to the exact position of the target. The ordering denoted by $\tilde{n}$ is depending on the numerical SVD of $\mathbb{K}^{c a v}$.

diagonal terms are equal to $S_{n n}\left(R_{\Omega}\right)=-J_{n}\left(k R_{\Omega}\right) / H_{n}\left(k R_{\Omega}\right)$. As the receivers and the emitters are on the same circle, and within the weak approximation, it turns out that

$$
\sigma_{n}^{\text {free }}=N\left|H_{n}\left(k R_{\Gamma}\right)\right|^{2} S_{n n}\left(k R_{\Omega}\right) \quad \text { and } \quad \sigma_{n}^{\text {cav }}=N\left|f_{n}\left(k R_{\Gamma}\right)\right|^{2} S_{n n}\left(k R_{\Omega}\right)
$$

It has been shown $[1,34,31,32]$ that the $S_{n n}$ coefficients are starting to decrease when $n$ is of the order of $\left|k R_{\Omega}\right|$. It can also be shown (see Appendix B) that, when $n$ tends to infinity, the $S_{n n}$ are decreasing faster than the coefficients $\left|H_{n}\left(k R_{\Gamma}\right)\right|^{2}$ or $\left|f_{n}\left(k R_{\Gamma}\right)\right|^{2}$ are eventually increasing (Figure 4). It is worth noticing, that in the cavity configuration, the oscillations in the singular spectrum are linked to the vicinity of $R_{\Sigma}$ with one of the zeroes of the associated $J_{n}$ Bessel function [26].

\section{Combining the singular system}

It is obvious that by combining the various back-propagated fields, it should be possible to derive some information on the target localization and shape. Various schemes are already available, as for example the DORT method, the Linear Sampling method (LSM), the MUSIC method, the Factorization method, ... 

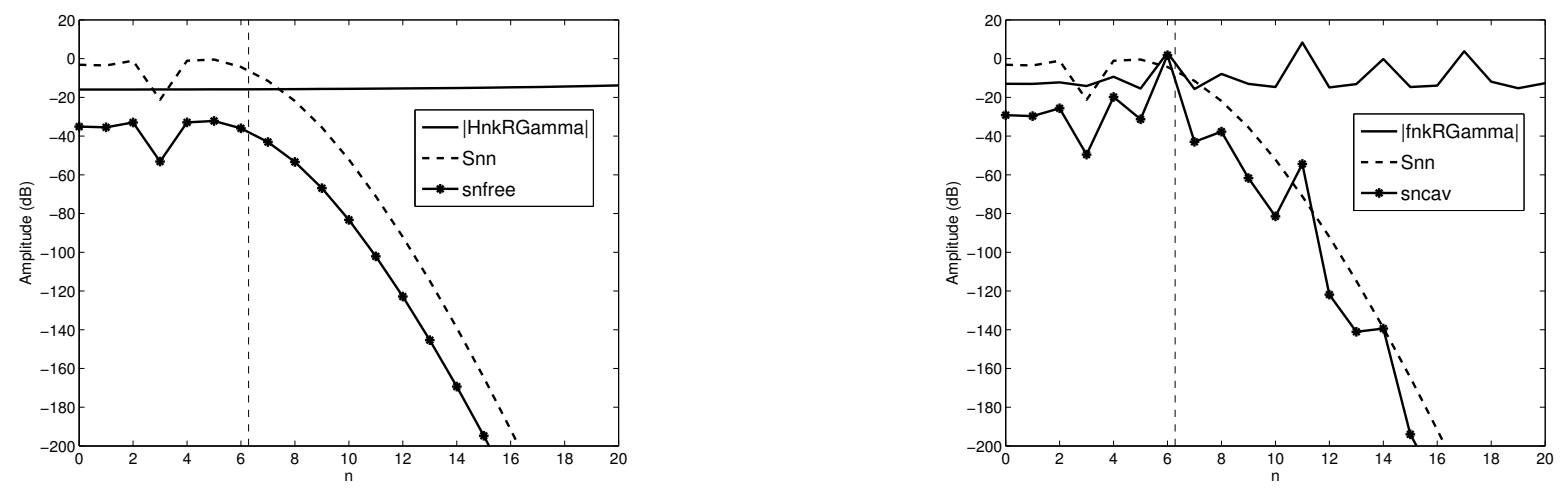

Figure 4. Evolution of the $\left|S_{n n}\right|$ parameters and the singular values $\left|\sigma_{n}\right|$ with respect to $n$, in the free-space case (left) or in the cavity (right). The measurement circle is at $R_{\Gamma}=4 \lambda$, the casing at $R_{\Sigma}=R_{\Gamma}+\lambda / 4$ and the metallic scatterer has a radius of $R_{\Omega}=\lambda$. The vertical line is marking the limit $n=k R_{\Omega}$ where the singular values are starting to decrease.

\subsection{A brief review of some available imaging methods}

As we already mentioned, for a point scatterer, there is a one-to-one correspondence between the singular vector corresponding to the leading singular value and the point scatterer location. The components of this singular vector give the complex amplitudes of the $N$ antennas which allows one to synthesize a cylindrical wave focusing onto the scatterer. The DORT indicator can then be written as

$$
I_{D O R T}(\vec{r} \in \Omega)=\left|\left\langle G(\vec{r} ;) \mid u_{n_{1}}\right\rangle_{\Gamma}\right|^{2}
$$

where $n_{1}$ is the index of the dominant singular value. If a numerical singular value decomposition (SVD) is employed, the dominant singular value is always the first one. If there exist three small size scatterers, well separated from each other, three dominant singular values can be observed, the index $n$ is the index associated to any of these three singular values. Unfortunately, the DORT method only deals with small size scatterers.

The Linear Sampling Method is exploiting the far-field equation in which the righthand-side term is a known analytic function as for example the Green function and the integral kernel is the measured field pattern. This method is based on the fact that an approximate solution of this far-field equation may exists whose norm will blow up to infinity for all points approaching the boundary of the scatterer from inside. As the linear system is ill-conditioned, a Tikhonov regularization method is applied to provide a regularized solution such that

$$
I_{L S M}(\vec{r} \in \Omega)=\sum_{n=1}^{N}\left(\frac{\sigma_{n}}{\sigma_{n}^{2}+\alpha}\right)^{2}\left|\left\langle G(\vec{r} ; \cdot) \mid u_{n}\right\rangle_{\Gamma}\right|^{2}
$$

Plotting the LSM indicator theoretically enables us to know if the sampling point belongs or not to the targets. The difficulty relies here in the correct tuning of the Tikhonov regularization coefficient $\alpha$, even if some mathematical rules may exist as for example the Morozov discrepancy principle. If $\alpha$ is too small, only the contribution from the targets 
exterior will be present. By decreasing $\alpha$, it is possible to retrieve the boundaries of the target. If $\alpha$ is correctly chosen, both the interior and the boundaries contributions are visible.

The Factorization method is similar to the Linear Sampling method, but instead of exploiting the far-field operator $\mathcal{F}$, it considers an integral kernel based on $\left[\mathcal{F F}^{*}\right]^{1 / 4}$. Again, a regularized solution is derived by considering the associated shape indicator

$$
I_{F M}(\vec{r} \in \Omega)=\sum_{n=1}^{N}\left(\frac{\sqrt{\sigma_{n}}}{\sigma_{n}+\alpha}\right)^{2}\left|\left\langle G(\vec{r} ; \cdot) \mid u_{n}\right\rangle_{\Gamma}\right|^{2}
$$

The same issues concerning the choice of the regularization parameter arise.

The imaging function for multiple signal classification (MUSIC) method is such that

$$
\left[I_{M U S I C}(\vec{r} \in \Omega)\right]^{-1}=\sum_{n=P}^{n=N}\left|\left\langle G(\vec{r} ;) \mid u_{n}\right\rangle_{\Gamma}\right|^{2}
$$

This method favors the contributions of the singular vectors which belong to the null space delimited by the index $P$. To implement the MUSIC method, the most important step is to determine the signal subspace dimension $P[10]$. For a detailed discussion of the determination of the number $P$, one may refer to $[33,17]$.

The imaging function for the subspace migration (SM) method proposed in [19] is such that

$$
I_{S M}(\vec{r} \in \Omega)=\sum_{N_{1} \leq n \leq N_{2}}\left|\left\langle G(\vec{r} ; \cdot) \mid u_{n}\right\rangle_{\Gamma}\right|^{2}
$$

where $N_{1} \leq n \leq N_{2}$ are selected such that the normalized singular values $\sigma_{n} / \sigma_{1}$ belong

to an interval $[a, b] \subset(0,1]$ which must be carefully chosen depending on the parts of the target that one wants to reconstruct.

\subsection{A combined expression}

By taking a closer look to the previous expressions, it is obvious that all these methods can be formalized into a single formula

$$
\left.I(\vec{r})=\sum_{n} h\left(\sigma_{n}\right)|\langle G(\vec{r} ; \cdot) ; \cdot)| u_{n}\right\rangle\left._{\Gamma}\right|^{2}
$$

where $h\left(\sigma_{n}\right)$ is a function which differs from one method to the other (Figure 5). Indeed, we have

$h_{\text {DORT }}(t, T)=\left\{\begin{array}{cc}1 & t=T \\ 0 & \text { otherwise }\end{array}, \quad h_{M U S I C}(t, S N R)=\left\{\begin{array}{ll}1 & t \leq S N R \\ 0 & \text { otherwise }\end{array}\right.\right.$,

$h_{L S M}(t, \alpha)=\left(\frac{t}{\alpha+t^{2}}\right)^{2}, \quad h_{F M}(t, \alpha)=\left(\frac{\sqrt{t}}{\alpha+t}\right)^{2}, \quad h_{S M}(t, a, b)= \begin{cases}1 & a<t<b \\ 0 & \text { otherwise }\end{cases}$

where T, $\alpha, S N R, a$ and $b$ are external tuning parameters. For the DORT method, $T$ corresponds to the maximum value that must be reached. For the MUSIC method, 

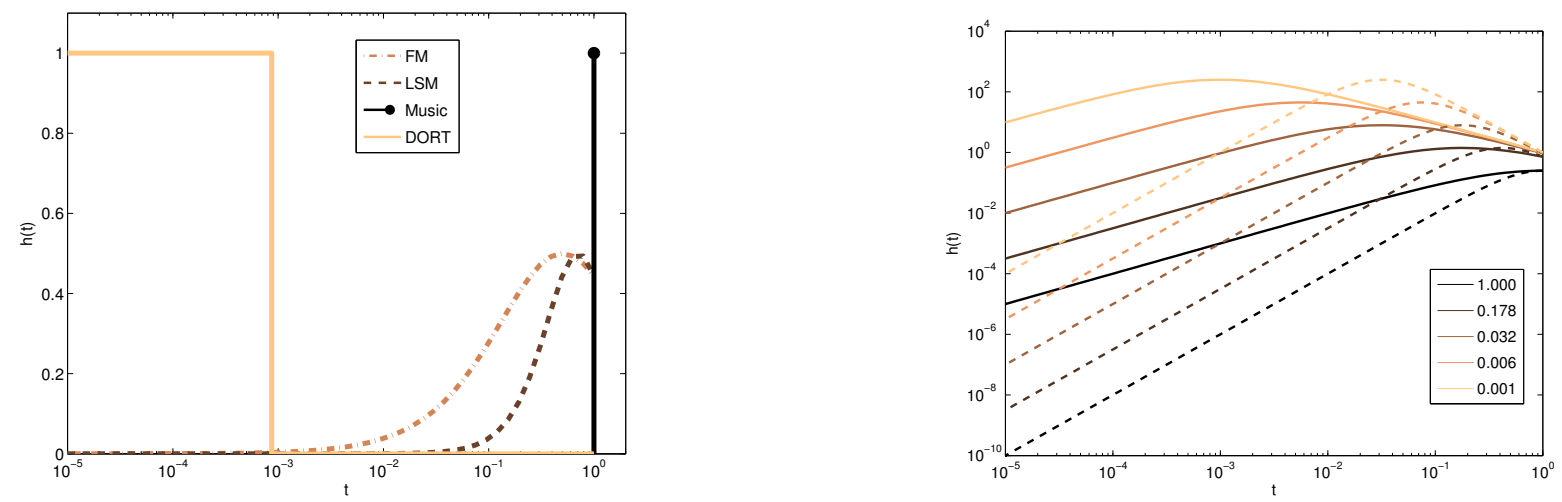

Figure 5. (Left) Weighting functions for the first five methods. (Right) Weighting functions for the LSM (dotted line) and the Factorization method (full line) for various values of the regularization parameter $\alpha$.

$S N R$ corresponds to the cut-off value for selecting the singular vectors which are part of the noise space. This cut-off value is usually based on the signal-to-noise ratio. The coefficient $\alpha$ is the regularization parameter which arises in the LSM or the Factorization method (Figure 5). It is of interest to note that, by playing with $\alpha$, we can favor the highest values of the input parameters $t$, or reversely favor the intermediate values, while in general the small values of the input parameter $t$ are neglected. This effect is more visibly on the Linear Sampling Method than on the Factorization Method. Finally, the choice of the interval $[a, b]$ will affect the capabilities of the shape indicator to detect either the inner parts, the boundary parts or the outer parts of the target.

\subsection{Extended-DORT method}

To overcome the aforementioned issues, we are providing another combination which is directly inspired from the DORT principle. Indeed, by naturally damping the singular vectors with their associated singular values, there is no need to truncate the summation neither to define some regularization parameters. The Extended-DORT (or EDORT) indicator is as follows

$$
I_{E D O R T}(\vec{r} \in \Omega)=\sum_{n}\left|\sigma_{n}\right|^{2}\left|\left\langle G(\vec{r} ; \cdot) \mid u_{n}\right\rangle_{\Gamma}\right|^{2}
$$

which corresponds to nothing but selecting $h_{E D O R T}(t)=t^{2}$. Hopefully, this EDORT indicator is a convergent series as demonstrated in Appendix $\mathrm{C}$ when the target is a centered circular metallic cylinder.

A similar expression, denoted as the reverse-time migration [17, 18], was derived by taking $h(t)=t$ and has been tested for small scatterers. For a single point scatterer, the singular values are all null except the first one and the summation is nothing but the classical DORT selection. Similarly, when $\alpha$ is becoming very large, the LSM indicator tends to the proposed EDORT indicator. 


\section{Results}

To investigate the rendering of the proposed methods, experimental scattered fields have been acquired within the microwave scanner developed at Institut Fresnel [6, 23, 24, 25].

\subsection{Measurement configuration and calibration protocol}

This scanner consists in a cylindrical cavity (Figure 6) entirely filled with water and enclosed by a metallic circular casing $\Sigma$ with radius $R_{\Sigma}=29.5 \mathrm{~cm}$ and center $O$. The incident electromagnetic fields are generated by an array of 64 biconical antennas acting sequentially as emitter and receiver and working at the single frequency of $434 \mathrm{MHz}$. These antennas are placed in the same horizontal plane, on a circle $\Gamma$ centered on $O$ and of slightly smaller radius than the casing $\left(R_{\Gamma}=27.6 \mathrm{~cm}\right)$ and are adapted to radiate within water with an estimated permittivity of $\varepsilon_{r w}=80.5+3.5 j$. Thanks to a multiplexer-de-multiplexer and a network analyzer, each antenna is selected as the emitting antenna, the 63 remaining antennas are then measuring the field, providing at the end a $63 \times 64$ multi-static measurement transfer matrix $\mathbb{K}^{\text {cav }}$.

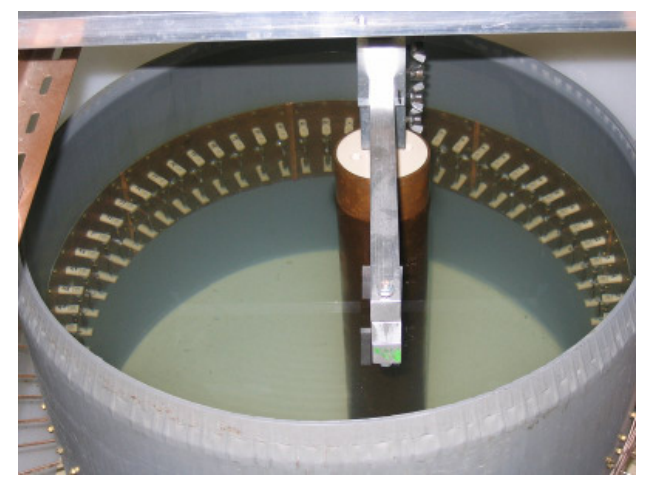

Figure 6. Picture of the microwave scanner measurement configuration currently exploited at Institut Fresnel

The measurement protocol is performed in four steps: (i) the incident field is measured with only water inside the tank, (ii) the total reference field is measured with a reference target positioned at the center of the setup, (iii) the total field is measured for the target under investigation, (iv) the water permittivity is measured by a coaxial open-ended probe. The reference target is a metallic circular cylinder, which has been chosen to be fully symmetrical and non dielectric in order not to be bothered by defining precisely its permittivity value.

The calibration is performed on the scattered fields, not on the total or incident fields. This calibration process, which is fully detailed in [23], takes advantages of the various symmetries (circular cavity, circular reference target). It enables to detect eventual defective antennas due to water corrosion at the soldered joints and create a binary mask which reflects the working antenna pairs. A complex calibration coefficients 

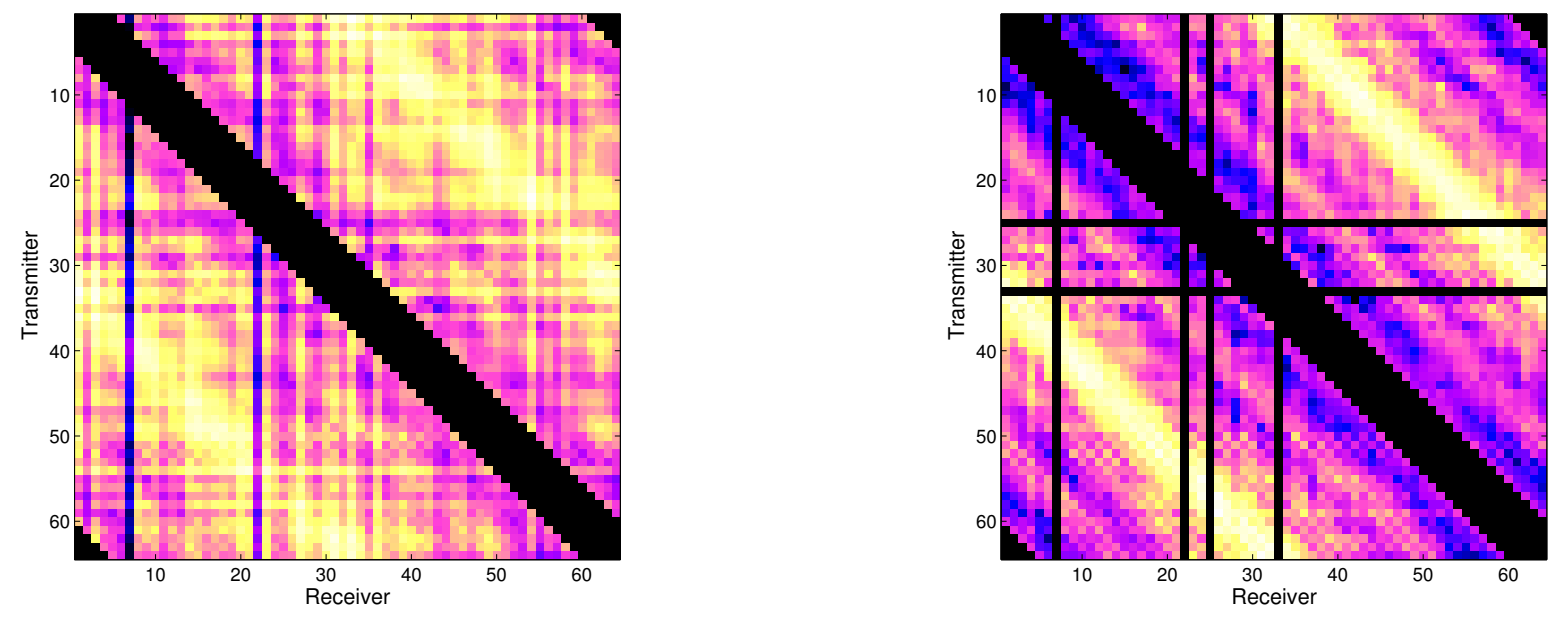

Figure 7. Multi-static matrix amplitude before calibration (left) and after calibration (right). The "black" lines/rows are corresponding to antenna pairs which have been detected as improperly working during the measurement process and which are removed from the calibration and the following imaging processing.

matrix is also computed by comparing the measured reference multi-static transfer matrix to the simulated one. Firstly, the emitting antennas are individually corrected in order that, in average, they convey the same level of signal and same phase reference. Secondly, the receiving antennas, that is the rows of the matrix, are individually corrected in order that, in average, they receive the same level of signal and the same phase. Of course, during the calibration process, the mask comes into play to neglect the defective antennas. The final calibration matrix (Figure 7) is then applied to the multi-static transfer matrix $\mathbb{K}^{c a v}$ of the unknown target, leading to transfer matrices which may eventually contain non-measured emitter/receiver pairs.

\subsection{Targets imaging}

Two types of metallic cylindrical bars (with circular or rectangular cross-section) are placed at different positions (centered and ex-centered) inside the cavity. The two cylinders present the same cross-section area, as the circular cylinder has a diameter of $4.5 \mathrm{~cm}$ while the rectangular cylinder has a $14 \times 4.5 \mathrm{~cm}$ rectangular cross-section.

Five of the shape indicator methods previously described have been applied on the same data sets and the reconstructed images are presented in Figure 8. The singular vectors of the multi-static transfer matrix have been computed with a numerical SVD. The Green's function inside the cavity has also been computed numerically with a 2D finite-element software $[6,24,35]$. The noise space range $P$ for the MUSIC indicator has been computed by performing a $-40 \mathrm{~dB}$ cutoff on the bandwidth of the scattered field with respect to the receiving angles to discriminate between the signal space and the noise space. The regularization parameter $\alpha$ was manually picked up. These parameters have unfortunately great influences on the reconstruction results for the FM, LSM or 
MUSIC indicators as shown in the movies which are available in the supplementary materials. Apart from the DORT method which fails at retrieving the target due to its relatively large size with respect to the wavelength, the other shape indicators are correctly detecting the targets. Some rings are also visible in the reconstructed images, due to the stationary waves which are created by the multiple reflections at the metallic boundaries. Finally, as pointed out in [15], dummy internal points which correspond to symmetrical points with respect to the target cross-section are also reconstructed by the various shapes indicators.

\section{Conclusion}

In this paper, we have proposed a new shape indicator which corresponds to the extension of the DORT method to the case of extended targets. It is still based on the singular value decomposition of the multi-static transfer matrix but it avoids the drawback of correctly selecting the hyper-parameters which are required in the MUSIC, the LSM or the Factorization methods. This Extended-DORT method has been tested against real data acquired in a microwave circular scanner and gives rather satisfying results when compared to the already cited methods. We also demonstrate the convergence of this type of indicator for a confined geometry or in a free space case for bi-dimensional configurations. These results are very promising and their extension to 3D environments will be tested in a near future.

\section{Acknowledgments}

The work of X.Y. Zhang was supported by a Chinese Scholarship Council - Ecole Centrale $\mathrm{PhD}$ grant.

\section{Appendix A. Derivation of the singular system of $\mathbb{K}^{\text {cav }}$ inside a metallic cavity}

In a circular metallic cavity, the multistatic scattering matrix can be written as

$$
\mathbb{K}^{\text {cav }}=\left[\mathbb{F}^{+}\left(\overrightarrow{O^{\prime} R}\right)\right]^{t} \mathbb{S}^{\prime} \mathbb{F}^{-}\left(\overrightarrow{O^{\prime} E}\right)
$$

where the propagation matrices $\mathbb{F}^{ \pm}$are defined in Eq. 6. In order to easily extract the singular vectors of $\mathbb{K}^{c a v}$ from these matrices, it is of interest to check if they are unitary matrices or not. To that end, we are analyzing the product $\left[\mathbb{F}^{ \pm}\left(\overrightarrow{O^{\prime} M}\right)\right]^{*} \mathbb{F}^{ \pm}\left(\overrightarrow{O^{\prime} M}\right)$,

$$
\begin{aligned}
{\left[\mathbb{F}^{ \pm}\left(\overrightarrow{O^{\prime} M}\right)\right]^{*} \mathbb{F}^{ \pm}\left(\overrightarrow{O^{\prime} M}\right) } & =\left[\mathbb{T}_{\mathbb{J}}^{ \pm}\left(\overrightarrow{O O^{\prime}}\right)^{-1} \mathbb{F}^{ \pm}(\overrightarrow{O M})\right]^{*} \mathbb{T}_{\mathbb{J}}^{ \pm}\left(\overrightarrow{O O^{\prime}}\right)^{-1} \mathbb{F}^{ \pm}\left(\overrightarrow{O^{\prime} M}\right) \\
& =\left[\mathbb{F}^{ \pm}(\overrightarrow{O M})\right]^{*}\left[\mathbb{T}^{ \pm} J\left(\overrightarrow{O O^{\prime}}\right) \mathbb{T}_{\mathbb{J}}^{ \pm}\left(\overrightarrow{O O^{\prime}}\right)^{*}\right]^{-1} \mathbb{F}^{ \pm}\left(\overrightarrow{O^{\prime} M}\right)
\end{aligned}
$$

When the emitters and the receivers are far from the target with respect to the wavelength, i.e., when employing a weak approximation, the term in $k R_{O^{\prime}}$ tends to 0 . As the matrix $\mathbb{T}_{\mathbb{J}}^{ \pm}\left(\overrightarrow{O O^{\prime}}\right)$ has a Toeplitz structure and as all the outer-diagonal terms 
are also tending to 0 except for the diagonal terms which are tending to 1 , we can approximate $\mathbb{T}_{\mathbb{J}}^{ \pm}\left(\overrightarrow{O O^{\prime \prime}}\right)$ by the identity matrix $\mathbb{I}_{d}$. We then have

$$
\left[\mathbb{F}^{ \pm}\left(\overrightarrow{O^{\prime} M}\right)\right]^{*} \mathbb{F}^{ \pm}\left(\overrightarrow{O^{\prime} M}\right) \approx\left[\mathbb{F}^{ \pm}(\overrightarrow{O M})\right]^{*} \mathbb{F}^{ \pm}(\overrightarrow{O M})
$$

It is therefore natural to consider the following term

$$
\begin{aligned}
{\left[\mathbb{F}^{ \pm}(\overrightarrow{O M})\left[\mathbb{F}^{ \pm}(\overrightarrow{O M})\right]^{*}\right]_{n m} } & =\sum_{p}\left[\mathbb{F}^{ \pm}(\overrightarrow{O M})\right]_{n p}\left[\mathbb{F}^{ \pm}(\overrightarrow{O M})\right]_{p m}^{*} \\
& =\sum_{p} f_{n}\left(k \rho_{p}\right) e^{ \pm j n \phi_{p}} \overline{f_{m}\left(k \rho_{p}\right) e^{ \pm j m \phi_{p}}} \\
& =\sum_{p} f_{n}\left(k \rho_{p}\right) f_{m}^{*}\left(k \rho_{p}\right) e^{ \pm j(n-m) \phi_{p}}
\end{aligned}
$$

In the present configuration, the receivers are all on the same circular probing line, with radius $R_{\Gamma}$. Therefore, the term

$$
f_{n}\left(k \rho_{p}\right) f_{m}^{*}\left(k \rho_{p}\right)=f_{n}\left(k R_{\Gamma}\right) f_{m}^{*}\left(k R_{\Gamma}\right)
$$

is only varying with respect to the indexes $n$ and $m$, which can help in simplifying the previous expressions. Indeed,

$$
\begin{aligned}
{\left[\mathbb{F}^{ \pm}(\overrightarrow{O M})\left[\mathbb{F}^{ \pm}(\overrightarrow{O M})\right]^{*}\right]_{n m} } & =f_{n}\left(k R_{\Gamma}\right) f_{m}^{*}\left(k R_{\Gamma}\right) \sum_{p} e^{ \pm j(n-m) \phi_{p}} \\
& =f_{n}\left(k R_{\Gamma}\right) f_{m}^{*}\left(k R_{\Gamma}\right) N \delta_{n, m}
\end{aligned}
$$

as the receivers are equally spaced on the circular probing line $\Gamma$. The propagation matrices can be scaled to produce unitary matrices. Indeed, the scaled propagation matrix $\mathbb{F}_{s}^{ \pm}(\overrightarrow{O M})=\left[\mathbb{D}^{\text {cav }}(\overrightarrow{O M})\right]^{-1 / 2} \mathbb{F}^{ \pm}(\overrightarrow{O M})$ with

$\mathbb{D}^{c a v}(\overrightarrow{O M})=\left(\begin{array}{ccc}\sum_{p}\left|f_{-n}\left(k \rho_{p}\right)\right|^{2} & 0 & 0 \\ 0 & \sum_{p}\left|f_{0}\left(k \rho_{p}\right)\right|^{2} & 0 \\ 0 & 0 & \sum_{p}\left|f_{n}\left(k \rho_{p}\right)\right|^{2}\end{array}\right)$

will be such that $\mathbb{F}_{s}^{ \pm}(\overrightarrow{O M})\left[\mathbb{F}_{s}^{ \pm}(\overrightarrow{O M})\right]^{*}=\mathbb{I}_{d}$. With this scaling, the multi-static scattering matrix can be written as

$$
\mathbb{K}^{c a v}=\left[\mathbb{F}_{s}^{+}(\overrightarrow{O R})\right]^{t}\left[\mathbb{D}^{c a v}(\overrightarrow{O R})\right]^{1 / 2} \mathbb{S}\left[\mathbb{D}^{c a v}(\overrightarrow{O E})\right]^{1 / 2} \mathbb{F}_{s}^{+}(\overrightarrow{O E})
$$

Identically, we will obtain

$$
\mathbb{K}^{c a v}=\left[\mathbb{F}_{s}^{+}\left(\overrightarrow{O^{\prime} R}\right)\right]^{t}\left[\mathbb{D}^{c a v}\left(\overrightarrow{O^{\prime} R}\right)\right]^{1 / 2} \mathbb{S}^{\prime}\left[\mathbb{D}^{c a v}\left(\overrightarrow{O^{\prime} E}\right)\right]^{1 / 2} \mathbb{F}_{s}^{+}\left(\overrightarrow{O^{\prime} E}\right)
$$

\section{Appendix B. Singular values asymptotic behavior}

For a circular target, when the receivers and the emitters are on the same circle, and within the weak approximation, the singular values of the multistatic scattering matrix are such that

$$
\sigma_{n}^{\text {free }}=N\left|H_{n}\left(k R_{\Gamma}\right)\right|^{2} S_{n n}\left(k R_{\Omega}\right) \quad \text { and } \quad \sigma_{n}^{\text {cav }}=N\left|f_{n}\left(k R_{\Gamma}\right)\right|^{2} S_{n n}\left(k R_{\Omega}\right)
$$


where $N$ corresponds to the number of emitters or receivers and $S_{n n}$ to the diagonal terms of the scattering matrix. When the target is a metallic one, the $S_{n n}$ coefficients are given by

$$
S_{n n}\left(k R_{\Omega}\right)=-\frac{J_{n}\left(k R_{\Omega}\right)}{H_{n}\left(k R_{\Omega}\right)}
$$

It is possible to derive asymptotic expressions when $n$ is large with respect to $\left|k R_{\Omega}\right|$. Indeed, it can be shown $[34,1,30,31,32]$ that

$$
\left|S_{n n}\left(k R_{\Omega}\right)\right| \sim \frac{1}{2}\left(\frac{e\left|k R_{\Omega}\right|}{2 n}\right)^{2 n}, \quad n \gg\left|k R_{\Omega}\right|, n \geq 1
$$

Appendix B.1. Free space configuration

The behavior of the terms in $k R_{\Gamma}$ will differ if $n$ is smaller or larger than $\left|k R_{\Gamma}\right|$. If $\left|k R_{\Gamma}\right|>n \gg\left|k R_{\Omega}\right|$, we can only apply the asymptotic formulas for large argument values with respect to $\left|k R_{\Gamma}\right|$. If $n \gg\left|k R_{\Gamma}\right|$, we can apply the asymptotic formulas for large index values with respect to $n$. We finally obtain

$$
\begin{array}{rlrl}
\left|\sigma_{n}^{\text {free }}\right| \sim \frac{N}{\pi\left|k R_{\Gamma}\right|}\left(\frac{e\left|k R_{\Omega}\right|}{2 n}\right)^{2 n}, & \text { if }\left|k R_{\Gamma}\right|>n \gg\left|k R_{\Omega}\right| \\
\left|\sigma_{n}^{\text {free }}\right| \sim \frac{N}{\pi n}\left(\frac{\left|k R_{\Omega}\right|}{\left|k R_{\Gamma}\right|}\right)^{2 n}, & & \text { if } n \gg\left|k R_{\Gamma}\right|>\left|k R_{\Omega}\right|
\end{array}
$$

In the two cases, i.e., when the measurement line is close or far from the target, we can see that $\left|\sigma_{n}^{\text {free }}\right| \rightarrow 0$ when $n \rightarrow \infty$.

\section{Appendix B.2. Cavity configuration}

In the cavity configuration, we also must take into account the terms in $k R_{\Sigma}$, keeping in mind that $R_{\Sigma}-R_{\Gamma}=\frac{\lambda}{4}$ and thus $\left|k R_{\Sigma}-k R_{\Gamma}\right|<2$. Thus, there are only two cases to consider: $\left|k R_{\Sigma}\right|>\left|k R_{\Gamma}\right|>n \gg\left|k R_{\Omega}\right|$ and $n \gg\left|k R_{\Sigma}\right|$. We obtain

$$
\begin{aligned}
\left|f_{n}\left(k R_{\Gamma}\right)\right|^{2} \sim \frac{2}{\pi\left|k R_{\Gamma}\right|} \frac{1}{\sin ^{2}\left(k R_{\Sigma}-n \frac{\pi}{2}-\frac{\pi}{4}\right)}, & \text { if }\left|k R_{\Gamma}\right|>n \gg\left|k R_{\Omega}\right| \\
\left|f_{n}\left(k R_{\Gamma}\right)\right|^{2} \sim \frac{2}{\pi n}\left[\left(\frac{\left|k R_{\Sigma}\right|}{\left|k R_{\Gamma}\right|}\right)^{n}-\left(\frac{\left|k R_{\Gamma}\right|}{\left|k R_{\Sigma}\right|}\right)^{n}\right]^{2}\left(\frac{2 n}{e\left|k R_{\Sigma}\right|}\right)^{2 n}, & \text { if } n \gg\left|k R_{\Sigma}\right|
\end{aligned}
$$

The asymptotic behavior of the singular values is thus given by

$$
\begin{aligned}
\left|\sigma_{n}^{c a v}\right| \sim \frac{N}{\pi\left|k R_{\Gamma}\right|}\left(\frac{e\left|k R_{\Omega}\right|}{2 n}\right)^{2 n} \frac{1}{\sin ^{2}\left(k R_{\Sigma}-n \frac{\pi}{2}-\frac{\pi}{4}\right)}, & \text { if }\left|k R_{\Gamma}\right|>n \gg\left|k R_{\Omega}\right| \\
\left|\sigma_{n}^{c a v}\right| \sim \frac{N}{\pi n}\left[\left(\frac{\left|k R_{\Omega}\right|}{\left|k R_{\Gamma}\right|}\right)^{n}-\left(\frac{\left|k R_{\Omega}\right|\left|k R_{\Gamma}\right|}{\left|k R_{\Sigma}\right|^{2}}\right)^{n}\right]^{2}, & \text { if } n \gg\left|k R_{\Sigma}\right|
\end{aligned}
$$

In the two cases, i.e., when the measurement line is close or far from the target, we can also conclude that $\left|\sigma_{n}^{c a v}\right| \rightarrow 0$ when $n \rightarrow \infty$. 


\section{Appendix C. EDORT summation behavior}

We are now considering the series which appears in the EDORT method (Eq. 25) and trying to figure out if it is convergent or not. To simplify the derivation, we will assume that the scatterer is a centered cylindrical metallic target.

\section{Appendix C.1. Free space configuration}

For a centered cylindrical target, the singular vectors are such that $u_{n}^{\text {free }}(\vec{r} \in \Gamma)=$ $d_{n}^{\text {free }} H_{n}\left(k R_{\Gamma}\right) e^{i n \theta_{r}}$ with $\left[d_{n}^{\text {free }}\right]^{-1}=\sqrt{N}\left|H_{n}\left(k R_{\Gamma}\right)\right|$ where $N$ is the number of receivers. The EDORT indicator is thus corresponding to

$$
I_{E D O R T}^{\text {free }}(\vec{r})=N^{3} \sum_{n}\left[S_{n n}\left|H_{n}\left(k R_{\Gamma}\right)\right|^{3}\right]^{2}\left|J_{n}(k \rho)\right|^{2}
$$

We denote the series term by $a_{n}^{\text {free }}(\vec{r})=\left[S_{n n}\left|H_{n}\left(k R_{\Gamma}\right)\right|^{3}\right]^{2}\left|J_{n}(k \rho)\right|^{2}$. For large orders $n$, it can be shown that

$$
a_{n}^{\text {free }}(\vec{r}) \sim\left(\frac{1}{\pi n}\right)^{4}\left(\frac{k R_{\Omega}}{k R_{\Gamma}}\right)^{4 n}\left(\frac{k \rho}{k R_{\Gamma}}\right)^{2 n}, \quad \text { if } n \gg 1
$$

In order to determine the convergence of the series, we exploit d'Alembert rule which states that, as $a_{n}^{\text {free }}$ are a sequence of positive terms, if the limit of $\left|a_{n+1}^{\text {free }} / a_{n}^{\text {free }}\right|$ when $n \rightarrow \infty$ is lower than 1 , than the series is convergent. We thus show that

$$
\left|\frac{a_{n+1}^{\text {free }}(\vec{r})}{a_{n}^{\text {free }}(\vec{r})}\right| \sim\left(\frac{n}{n+1}\right)^{4}\left(\frac{k R_{\Omega}}{k R_{\Gamma}}\right)^{4}\left(\frac{k \rho}{k R_{\Gamma}}\right)^{2}, \quad \text { if } n \gg 1
$$

For any evaluation point $\vec{r}$ which is located inside the circle delimited by the probing line $\Gamma$, when $n \rightarrow \infty$, this ratio tends to 0 . The series is therefore convergent.

\section{Appendix C.2. Cavity configuration}

For a centered cylindrical target, the singular vectors are such that $u_{n}^{c a v}(\vec{r} \in \Gamma)=$ $d_{n}^{\text {cav }} f_{n}\left(k R_{\Gamma}\right) e^{i n \theta_{r}}$ with $\left[d_{n}^{c a v}\right]^{-1}=\sqrt{N}\left|f_{n}\left(k R_{\Gamma}\right)\right|$ where $N$ is the number of receivers. The EDORT indicator is thus corresponding to

$$
I_{E D O R T}^{c a v}(\vec{r})=N^{3} \sum_{n}\left[S_{n n}\left|f_{n}\left(k R_{\Gamma}\right)\right|^{3}\right]^{2}\left|J_{n}(k \rho)\right|^{2}
$$

We denote the series term by $a_{n}^{\text {cav }}(\vec{r})=\left[S_{n n}\left|f_{n}\left(k R_{\Gamma}\right)\right|^{3}\right]^{2}\left|J_{n}(k \rho)\right|^{2}$. For large orders $n$, we obtain

$a_{n}^{c a v}(\vec{r}) \sim\left(\frac{1}{\pi n}\right)^{4}\left(\frac{k R_{\Omega}}{k R_{\Sigma}}\right)^{4 n}\left[\left(\frac{k R_{\Sigma}}{k R_{\Gamma}}\right)^{n}-\left(\frac{k R_{\Gamma}}{k R_{\Sigma}}\right)^{n}\right]^{6}\left(\frac{k \rho}{k R_{\Sigma}}\right)^{2 n}, \quad$ if $n \gg 1$

We now look at the ratio between two adjacent terms

$$
\left|\frac{a_{n+1}^{c a v}(\vec{r})}{a_{n}^{c a v}(\vec{r})}\right| \sim\left(\frac{n}{n+1}\right)^{4}\left(\frac{k R_{\Omega}}{k R_{\Sigma}}\right)^{4}\left(\frac{k R_{\Gamma}}{k R_{\Sigma}}\right)^{6}\left[\frac{1-\left(\frac{k R_{\Gamma}}{k R_{\Sigma}}\right)^{2(n+1)}}{1-\left(\frac{k R_{\Gamma}}{k R_{\Sigma}}\right)^{2 n}}\right]^{6}\left(\frac{k \rho}{k R_{\Sigma}}\right)^{2}
$$

When $n \rightarrow \infty$, this ratio is tending towards 0 , thus the summation is converging. 
[1] D. Colton and R. Kress. Inverse Acoustic and electromagnetic scattering theory. Springer, Berlin, 1992.

[2] R. Kleinman and P.M. van den Berg. A modified gradient method for two-dimensional problems in tomography. J. Comput. Appl. Math., 42:17-35, 1992.

[3] P.M. van den Berg and R. Kleinman. A constrast source inversion method. Inverse Problems, 13:1607-1620, 1997.

[4] T. Isernia, L. Crocco, and M. D'Urso. New tools and series for scattering problems in lossy media. IEEE Geosci. Remote Sens. Lett., 1:327-331, 2004.

[5] R. Marklein, L. Balasubramanian, A. Qing, and K.J. Langenberg. Linear and nonlinear iterative scalar inversion of multi-frequency multi-bistatic experimental electromagnetic scattering data. Inverse Problems, 17(6):1597-1610, 2001.

[6] R. Lencrerot, A. Litman, H. Tortel, and J.-M. Geffrin. Imposing zernike representation for twodimensional targets imaging. Inverse Problems, 25:035012, 2009.

[7] A. Abubakar and P.M. van den Berg. The contrast source inversion method for location and shape reconstruction. Inverse Problems, 18:495-510, 2002.

[8] A. Kak and M. Slaney. Principles of Computerized Tomographic Imaging. SIAM, 2001.

[9] D. Colton, J. Coyle, and P. Monk. Recent developments in inverse acoustic scattering theory. SIAM Review, 42(3):369-414, 2000.

[10] M. Cheney. The linear sampling method and the music algorithm. Inverse Problems, 17:591-595, 2001.

[11] A. Tacchino, J. Coyle, and M. Piana. Numerical validation of the linear sampling method. Inverse Problems, 18:511-527, 2002.

[12] A. Kirsch. The music algorithm and the factorization method in inverse scattering theory for inhomogeneous media. Inverse Problems, 18:1025-1040, 2002.

[13] H. Ammari, E. Iakovleva, and D. Lesselier. A music algorithm for locating small inclusions buried in a half space from the scattering amplitude at a fixed frequency. SIAM Multiscale Modelling simul., 3:597-628, 2005.

[14] R. Potthast. A survey on sampling and probe methods for inverse problems. Inverse Problems, 22(2):R1-R47, 2006.

[15] I. Catapano, L. Crocco, and T. Isernia. On simple methods for shape reconstruction of unknown scatterers. IEEE Trans. Antennas Propagat., 55(5):1431-1436, 2007.

[16] H. Ammari, J. Garnier, V. Jugnon, and H. Kang. Direct reconstruction methods in ultrasound imaging of small anomalies, volume 2035 of Lecture Notes in Mathematics, pages 31-56. Springer-Verlag, 2011.

[17] H. Ammari, J. Garnier, H. Kang, W.K. Park, and K. Solna. Imaging schemes for perfectly conducting cracks. SIAM Journal on Applied Mathematics, 71:68-91, 2011.

[18] H. Ammari, J. Garnier, and K. Solna. A statistical approach to optimal target detection and localization in the presence of noise. Waves in Random and Complex Media, 22:40-65, 2012.

[19] L. Borcea, G. Papanicolaou, and F. G. Vasquez. Edge illumination and imaging of extended reflectors. SIAM J. Imaging Sciences, 1:75-114, 2008.

[20] H. Tortel, G. Micolau, and M. Saillard. Decomposition of the time reversal operator for electromagnetic scattering. Journal of electromagnetic waves and applications, 13(5):687-719, 1999.

[21] K. Ito, B. Jin, and J. Zou. A direct sampling method to an inverse medium scattering problem. Inverse Problems, 28:025003, 2012.

[22] J-M. Geffrin, P. Sabouroux, and C. Eyraud. Free space experimental scattering database continuation: experimental set-up and measurement precision. Inverse Problems, 21:S117-S130, 2005 .

[23] A. Litman, J.-M. Geffrin, and H. Tortel. On the calibration of a multistatic scattering matrix measured by a fixed circular array of antennas. Progress in Electromagnetics Research, 110:1$21,2010$. 
[24] R. Lencrerot, A. Litman, H. Tortel, and J.-M. Geffrin. Measurement strategies for a confined microwave circular scanner. Inverse Problems in Science and Engineering, page 357869, 2009.

[25] A. Litman, R. Lencrerot, and J.-M. Geffrin. Combining spatial support information and shapebased method for tomographic imaging inside a microwave cylindrical scanner. Inverse Problems in Science and Engineering, 18:19-34, 2010.

[26] L. Crocco and A. Litman. On embedded microwave imaging systems: retrievable information and design guidelines. Inverse Problems, 25:065001, 2009.

[27] G. Micolau. Etude théorique et numérique de la méthode de la décomposition de l'opérateur de retournement temporel en diffraction électromagnétique. $\mathrm{PhD}$ thesis, Université Aix-Marseille, Marseille, France, 2001.

[28] J.-M. Geffrin. Imagerie microonde: Etude d'un scanner a $434 \mathrm{MHz}$ pour des applications biomedicales. PhD thesis, Univ. Paris XI, Orsay, France, 1995.

[29] P. M. van den Berg and J. T. Fokkema. Removal of undesired wavefields related to the casing of a microwave scanner. IEEE Trans. Microwave Theory and Tech., 51:187-192, 2003.

[30] M. Abramowitz and I. Stegun. Handbook of mathematical functions. Dover, New York, 1972.

[31] J.-G. Minonzio. Décomposition de l'Opérateur de Retournement Temporel appliqué à l'imagerie et à la caractérisation ultrasonore. PhD thesis, Université Paris 7, Paris, France, 2006.

[32] J.-G. Minonzio, F. Philippe, C. Prada, and M. Fink. Characterization of an elastic cylinder and an elastic sphere with the time-reversal operator: application to the sub-resolution limit. Inverse Problems, 24:025014, 2008.

[33] S. Hou, K. Solna, and H. Zhao. A direct imaging algorithm for extended targets. Inverse Problems, $22: 1151-1178,2006$.

[34] J.A. Stratton. Electromagnetic theory. McGraw-Hill, 1941.

[35] X. Zhang, H. Tortel, S. Ruy, and A. Litman. Microwave imaging of soil water diffusion using the linear sampling method. IEEE Geoscience and Remote Sensing Letters, 8:421-425, 2011. 


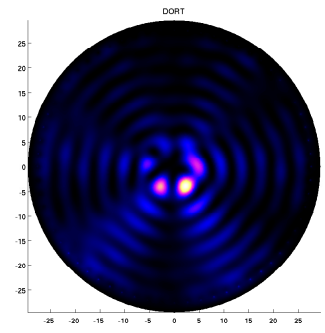

DORT

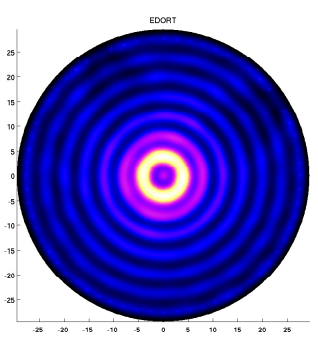

EDORT

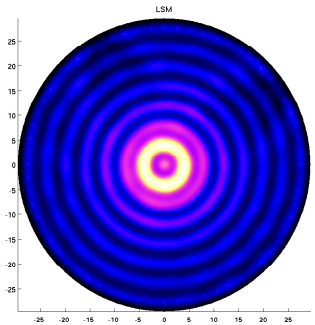

LSM

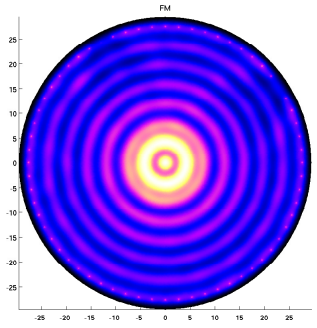

FM

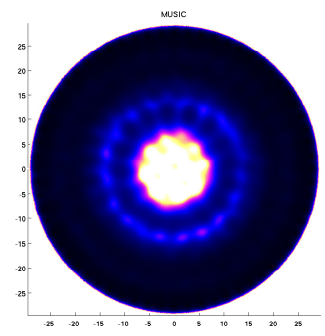

MUSIC

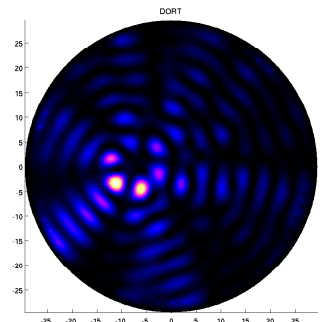

DORT

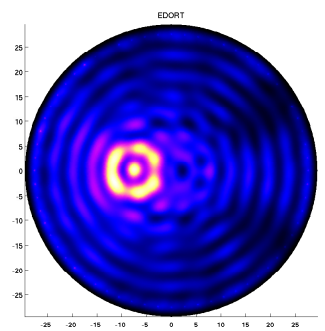

EDORT

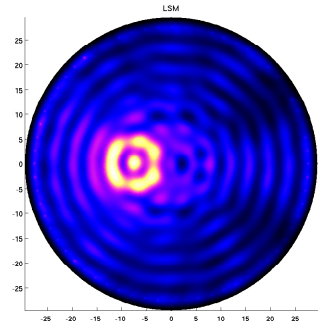

LSM

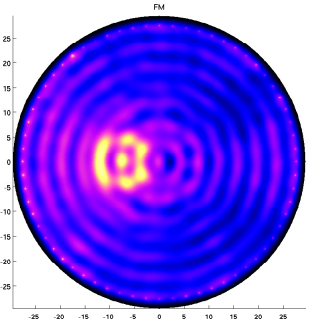

FM

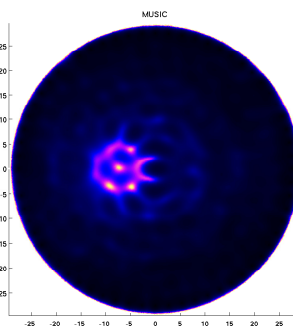

MUSIC

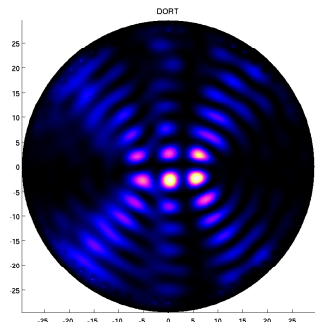

DORT

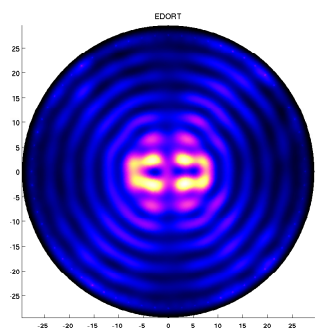

EDORT

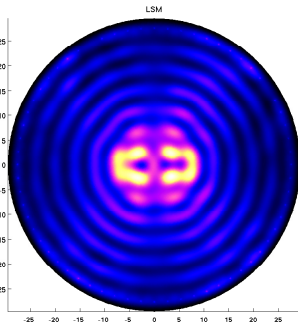

LSM

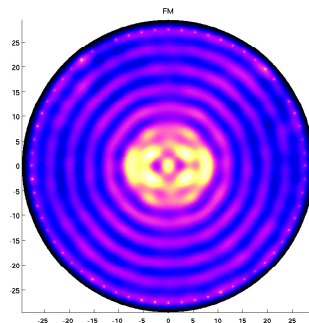

FM

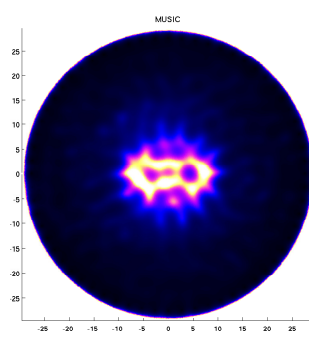

MUSIC

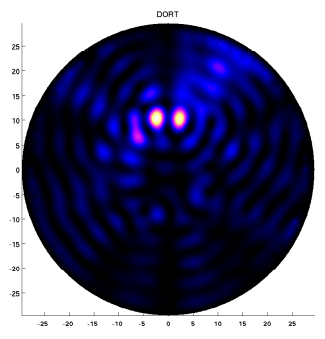

DORT

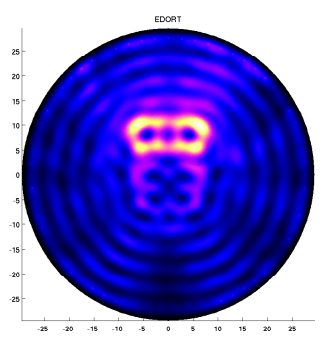

EDORT

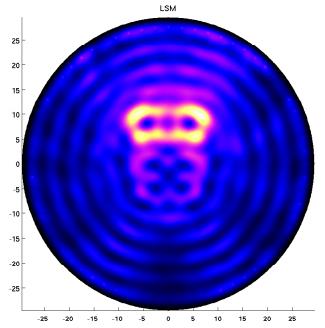

LSM

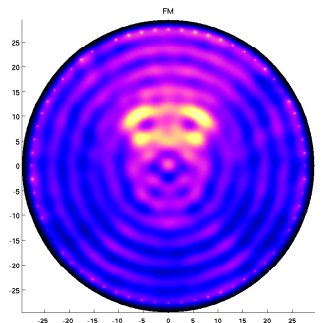

FM

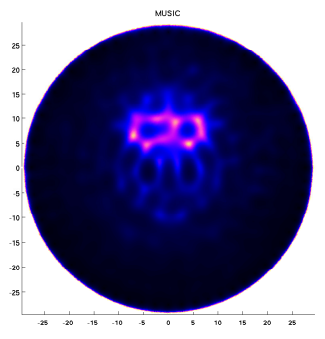

MUSIC

Figure 8. Reconstructions using the various shape indicators of the metallic cylindrical scatterers. (First column) Centered and circular cross-section. (Second column) Ex-centered and circular cross-section. (Third column) Centered and rectangular cross-section. (Fourth column) Ex-centered and rectangular cross-section. 